\title{
TAXONOMIC INDEX
}

Abyssoninoe hibernica

Abyssoninoe scopa

Acanthaster planci

Acrocirridae

Aglaophamus uruguayi

Aglaothamnion

Aglaothamnion sepositum

Alentia gelatinosa

Alitta brandti

Alitta grandis

Alitta succinea

Alitta virens

Amaena

Amblyosyllis madeirensis

Ammotrypane

Ammotrypane aulogaster

Ammotrypane aulopyge

Ammotrypane breviata

Ammotrypane cylindricaudatus

Ammotrypane nematoides

Ammotrypane scaphigera

Ammotrypane setigera

Ammotrypane syringopyge

Ampharete

Ampharete acutifrons

Amphicteis gunneri

Amphiduropsis

Amphiduros

Amphiglena mediterranea

Amphinomidae

Amphitrite

Ancistrosyllis groenlandica

Ancistrosyllis hamata

Anemonia viridis

Anobothrus gracilis

Anthogorgia bocki

Aonides

Aonides auricularis

Aonides californiensis

Aonides glandulosa

Aonides mayaguezensis

Aonides oxycephala

Aonides paucibranchiata

Aonides selvagensis

Aonides trifida

Aphelochaeta

Aphelochaeta

Aphrodita aculeata
20,25

17, 19-20

314,316

200

$249,252,255$

227

116

265

28

28

28

28

273

228

101-102, 104-108

104

107

105

106

106

106

107

107

252-253

203

203

164

164

$179,181-184$,

220-222, 280

200,245

273,276

199

200

227

265

12

59-64

60

59,63

59,63

59,63

$59-61,63-64$

$59,62-63$

$59,61-63$

59,63

45,73

326

266
Aphrodita longicornis

252

Aphroditella alta 252

Aponuphis bilineata

Aponuphis brementi

265

Aponuphis grubei

Arenicola marina

Arichlidon reysi

Aricidea

Aricidea annae

Aricidea capensis bansei

Aricidea catherinae

Aricidea claudiae

Aricidea fragilis mediterranea

Aricidea monicae

Aricidea pigmentata

Aricidea simonae

Aricidea simplex

Aricidea suecica meridionalis

Armandia cirrosa

Armandia loboi

Armandia polyophthalma

Ascidia mentula

Ascidiella aspersa

Asetocalamyzas laonicola

Asparagopsis

Asterias rubens

Augeneria aff. tentaculata

Augeneria algida

Augeneria polytentaculata

Augeneria riojai

Autolytinae

Autolytus alexandri

Autolytus brachycephalus

Autolytus edwarsi

Autolytus prolifer

Autolytus quindecimdentatus

Axionice

Axiothella

$201,203,265$

265

315-317

203

54, 243-244, 247, 326

265

199

199

201, 203-204

199, 201

265

$169,172-173$

199

54

199

222

252,256

265

315-316

315-316

343-350

227

315

252,256

$17,20,25$

21

20-21

228,233

265

228-229, 232, 265

228-229, 232

228

228

274

75-77

$\begin{array}{ll}\text { Baffinia } & 274 \\ \text { Bifurcaria bifurcata } & 227\end{array}$

Bispira melanostigmata $\quad 349$

Boccardia polybranchia

187, 191, 193-195

165

315

284

Botrillus schloseri

326-327

Branchiomma luctuosum

228,233

344,349 
Calanus finmarchicus

Calliblepharis ciliata

Capitella

Capitella capitata

Capitellidae

Capricornia

Cauleriella

Cauleriella alata

Cauleriella bioculata

Cauleriella venefica

Cenogenus abyssalis

Cenogenus descendens

Cenogenus eliae

Cenogenus nagae

Cenogenus simpla

Ceramium diaphanum

Ceratonereis brunensis

Ceratonereis costae

Cetorhinus maximus

Chaetopteridae

Chaetozone

Chaetozone allanotai

Chaetozone anasimus

Chaetozone armata

Chaetozone bansei

Chaetozone brunnea

Chaetozone commonalis

Chaetozone corona

Chaetozone curvata

Chaetozone diodonta

Chaetozone gibber

Chaetozone hystricosus

Chaetozone lunula

Chaetozone palaea

Chaetozone setosa

Chaetozone spinosa

Challis (Equichlamys) bifrons

Chloeia venusta

Chone arenicola

Chone collaris

Chone duneri

Chone filicaudata

Chone longiseta

Chrysopetalidae

Chrysopetalum debile

Chthamalus stellatus

Cirratulidae
187, 191, 193-197, 200-205,

220-223

243,245

158-160, 164-165

45, 55-56, 64-67, 71-73

$187,191,193,195,220-222$

$181,220,222$

66-67, 71, 73

87

82

81-83, 86-87, 89

87

87

181

243-247

265

116

35-36, 42-44

45-47, 51, 54-57, 65-66, 70,

72-73, 201, 20-204, 326

45-47, 51-56

$67-68,70-73$

66

73

45-47, 54-56

$51,56,68,73$

56

$51,56,73$

70-73

222

68-73

56,72

45-46, 48-51, 55-56

45, 51, 55-57, 65-66,

$70-73,219-222$

45-46, 48, 52-56

314

200

199

$181,201,203$

181

201

199

181

181

226
Cirratulus

Cirriformia cf. tentaculata

Cirrophorus branchiatus

Cirrophorus furcatus

Cistenides ehlersi

Claparedepelogenia inclusa

Claviderma

Clymene torquata

Clymenella fauchaldi

Clymenura

Clymenura clypeata

Clymenura tricirrata

Codium tomentosum

Conferva seposita

Corallina

Corallina elongata

Coryphaenoides rupestris

Cossura pygodactylata

Cossura soyeri

Cossuridae

Cucumaria frondosa

Cymodocea nodosa

Cystoseira baccata

Dasycladus vermicularis

Dentalia

Dentalium

Dindymenides

Dindymenides concina

Diopatra

Diopatra aciculata

Diopatra neapolitana

Diopatra viridis

Ditrupa arietina

Dorvillea rubrovittata

Dorvilleidae

Drilonereis

Dyctiopterus

Dyctiota

Eclysippe vanellii

Ehlersia ferruginea

Eisenia bicyclis

Eteone picta

Euchone rosea

Euchone southerni

Euclymene oerstedi

Euclymene palermitana

Eulalia mustela

Eunereis longissima
187, 191, 193, 195

201

201

199

252

265

48

75-76, 79

176

220

76-77

199

227

116

226

181,226

116

217, 220-224

200-201, 203

200

116

59-61, 63

227

181

120

120

108-109

108

252

331-333, 337-341

221,288

252-253

203

181

$151,155,181$

252

227

227

265

181

143

222

265

200

75-76, 78-79, 201,

221-222, 265

265

265

149, 200 
Eunice (Nicidion) cariboea

Eunice (Palola) siciliensis

Eunice aequalis

Eunice antennata

Eunice aphroditois

Eunice argentinensis

Eunice aucklandica

Eunice cariboea

Eunice elseyi

Eunice filamentosa

Eunice harassi

Eunice havaica

Eunice magellanica

Eunice martensi

Eunice miurai

Eunice norvegica

Eunice romanvivesi

Eunice rubra

Eunice siciliensis

Eunice torquata

Eunice unifrons

Eunice vittata

Eunicidae

Eupanthalis kinbergi

Euphrosine foliosa

Eupolymnia

Euprosinidae

Eurysyllis tuberculata

Eusyllinae

Eusyllis assimilis

Euzonus

Exogone dispar

Exogone hebes

Exogone naidina 181-184, 219-222, 228-229, 232

Exogone verugera

Exogoninae

Fabricia sabella

Fabricia stellaris

Flabelligera

Fucus hyperboreus

Fucus oederi

Galeolaria caespitosa

Gattyana cirrhosa

Gelidium sesquipedale

Glycera americana

Glycera capitata

Glycera dibranchiata

Glycera fallax

Glycera gigantea
$228,229,232$

91,92

91

252-253

95

91-92

95

92-93

332

95

252

95

95

$91,116,119$

93-95

91

98

265

91,95

181,201

$181,301-302,308$

265

200

273

200

203, 228

228

228

101

181

219, 221-222

181

349

349

$179,181-184$

326

116

116

309-317

265

227

252-253

222

287,289

265

222
Glycera tridactyla

Glycera unicornis

Goniada littorea

Goniada peruana

Goniadidae

Gymnonereidinae

Gyptis propinqua

Haliotis laevigata

Haliotis tuberculata

Haplosyllis anthogorgicola

Haplosyllis basticola

Haplosyllis crassicirrata

Haplosyllis spongicola

Harmothoe antilopes

Hediste diversicolor

Hesiocaeca

Hesiocaeca

Hesiodira

Hesione

Hesionella

Hesionidae

Hesionides

Hesionides arenaria

Hesiospina

Heteromastus filiformis

Heteropodarke

Hipponoe gaudichaudi

Holothuria frondosa

Holothuria tremula

Hydroides

Hydroides elegans

Hydroides norvegica' / H. norvegicus

$116-117,122-123$

Hydroides norvergica

115-116, 118-123

Hydroides norvergicum

Ianthella basta

11,16

Idanthyrsus armatus

Inermonephtys inermis

252

199

Jania rubens

181

Jasmineira

348

Johnstonia duplicata

76-77

Kesun

101-102, 108-109

Kesun abyssorum

109

Kesun fusus

108

Kuwaita

82

Lacydonia laureci

265

Lacydonidae 
Ladice

Lagisca

Laminaria

Laminaria hyperborea

Laminaria ochroleuca

Lanice conchilega

Laonice cirrata

Laphania

Laternula elliptica

Leaena

Leiocapitella glabra

Leitoscoloplos kerguelenis chilensis

Leocrates

Leocratides

Leodice panamena

Leodice unifrons

Leonnates

Leonnates persicus

Lepidonotus clava

Lepidonotus squamatus

Levinsenia flava

Levinsenia gracilis

Lizardia

Lopadorhynchus brevis

Lopadorhynchus henseni

Lopadorhynchus nationalis

Lophelia pertusa

Lumbricalus aotearoae

Lumbricalus campoyi

Lumbricalus composita

Lumbricalus harrisae

Lumbrineridae

Lumbrinerides amoureuxi

Lumbrineriopsis mucronata

Lumbrineriopsis paradoxa

Lumbrineris

Lumbrineris aniara

Lumbrineris futilis

Lumbrineris gracilis

Lumbrineris latreilli

Lumbrineris nonatoi

Lumbrineris scopa

Lumbrineris tetraura

Lygdamis muratus

Lysarete brasiliensis

Lysidice

Lysidice collaris

Lysidice ninetta

Macellicephala laubieri

Macrochaeta clavicornis
104

252-253

227, 233

116

227

201, 203

344

274

314-315, 317

274

199

176

164

164

93

95

28

204, 207

265

265

48

201, 204

164

212-213

212-213

212-213

116-118, 122

23

21-23

23

23

$81,83,85,87,89-90$

$17,23,25,199$

252

199

201,252

17,23

17,24

201, 203-204

24, 201, 203

199

19

191

199

252, 301-307

301-302, 306-307

205, 301-308

$91,95,181$

265

200
Madrepora pertusa

Maea

Magelona

Magelona agoensis

Magelona alleni

Magelona berkeleyi

Magelona californica

Magelona capax

Magelona capensis

Magelona cepiceps

Magelona cincta

Magelona conversa

Magelona cornuta

Magelona crenulifrons

Magelona equilamellae

Magelona falcifera

Magelona filiformis

Magelona filiformis

Magelona gemmata

Magelona heteropoda

Magelona japonica

Magelona japonica var. koreana

Magelona johnstoni

Magelona kamala

Magelona koreana

Magelona lenticulata

Magelona longicornis

Magelona magnahamata

Magelona mahensis

Magelona methae

Magelona mickminni

Magelona minuta

Magelona noppi

Magelona obockensi

Magelona pacifica

Magelona papillicornis

Magelona pectinata

Magelona petersenae

Magelona pettiboneae

Magelona phyllisae

Magelona pitelkai

Magelona polydentata

Magelona puchella

Magelona pygmaea

Magelona riojai

Magelona rosea

Magelona sachalinensis

Magelona symmetrica

Magelona wilsoni

Mahesia

Malacoceros
116-119

127

125-129, 131-137

126

128,137

133,137

126,135

131

126,133

$125,131-132,136$

$126,136-137$

125-126, 136

126, 128

126

128

125-126, 136

221-222, 265

199

125-126, 133, 136

126

126, 128

128

220

126, 133

126

126,133

126,137

135-136

$125,129,131,136$

126

126

135, 201

126

126

133

126, 135

126

126

135

$169,172-173$

126, 137

128

126

125-126, 133-136

252,256

126

126

$125,127-128,136$

265

164

176 
Malacoceros fuliginosus

Maldane sarsi

Malmgreniella arenicolae

Malmgreniella marphysae

Malmgreniella polypapillata

Marphysa amadae

Marphysa aransensis

Marphysa escobarae

Marphysa longula

Marphysa minima

Marphysa posterobranchiata

Marphysa regalis

Marphysa sanguinea

Marphysa veracruzensis

Mediomastus

Melinna palmata

Mesochaetopterus

Mesochaetopterus minuta

Mesochaetopterus minutus

Mesochaetopterus sagittarius

Mesophyllum

Mesophyllum lichenoides

Metasychis disparidentata

Metasychis gotio

Micronephtys mariae

Microphthalmus

Micropodarke

Monoculus finmarchicus

Monticellina

Monticellina

Monticellina dorsobranchialis

Monticellina heterochaeta

Myrianida pinnigera

Mytilus edulis

Nacella concina

Namalycastis

Neanthes

Neanthes arenaceodentata

Neanthes bongcoi

Neanthes caudata

Neanthes chilkaensis

Neanthes cricognatha

Neanthes meggitti

Neanthes philippinensis

Neanthes rubicunda

Neanthes succinea

Neanthes virens

Nematonereis unicornis

Neoamphitrite
199

91

91,95

98

96

91

98

98

91, 203, 288

96-98

243-244

201,221

35-44

37,39

$37,41,43$

41,43

$227,231,232,233$

226, 227

75-77

205

201

164

165

116

73

$46,73,243-244,247$

$199,205,222$

199, 205

228

314

314-315, 317

291

27-28

28

27-30

$28,145,149-150$

30

28,30

27-28, 20-33

30

$145,147,149-150$

187, 191, 193, 194-195,

200, 252

265

91, 98, 301-308

273-274
Neoamphitrite edwardsi

265

Neoamphitrite figulus

265

Neoleprea

Neonotomastus glabrus

Nephtys

Nephtys ferruginea

Nephtys hombergi

Nephtys incisa

Nepthys magellanica

Nereididae

Nereidinae

Nereimyra

Nereis diversicolor

Nereis lamellosa

Nereis madrepora pertusa

Nereis norvegica

Nereis rava

Nereis trifasciata

Nereis virens

Nerine oxycephala

Nicolea

Ninoe

Ninoe armoricana

Ninoe brasiliensis

Ninoe chilensis

Ninoe dolichognatha

Ninoe falklandica

Ninoe foliosa

Ninoe fusca

Ninoe fuscoides

Ninoe gemmea

Ninoe jessicae

Ninoe longibranchia

Ninoe marthae

Ninoe moorei

Ninoe oculata

Ninoe palmata

Notaulax nudicollis

Notomastus latericius

Octobranchus lingulatus

Odontosyllis ctenostoma

Omaria

Omphalopomopsis fimbriata

Onuphidae

Onuphis

Onuphis aff. eremita

Onuphis tenuis

Ophelia

Ophelia algida
273

48

252

172

205

201

176

$27-28,33,145,147,149-150$,

$181,243,245,248$

28,33

165

288, 291-292, 349

145,149

116-117, 119

116

145,149

242-243, 245-247

315

60

273-274

252

$17,24,25,199,203$

81,252

$81-82,84,86$

82

252

$81-82,84,86$

81-82

81-82

81-82, 84

81, 83-84, 86, 89

$81-82,84$

$81,83,85-86,89$

81-82

81

84

348

266

199

$181,228,230,232$

104

265

337-338, 341

252

252

252-253

101-104, 113

101-103 
Ophelia bicornis

Ophelia bicornis bicornis

Ophelia bipartita

Ophelia limacina

Ophelia praetiosa

Opheliidae

Opheliinae

Ophelina

Ophelina scaphigera

Ophelina acuminata

Ophelina breviata

Ophelina cylindricaudata

Ophelina gymnopyge

Ophelina nematoides

Ophelina pallidus

Ophelina setigera

Ophelina syringopyge

Opheliniinae

Ophiodromos

Ophiodromus pallidus

Ophryotrocha

Ophryotrocha geryonicola

Ophryotrocha isochaeta

Ophryotrocha labronica

Orbiniidae

Oriopsis

Osmundea oederi

Owenia

Owenia collaris

Owenia fusiformis

Padina pavonica

Palola siciliensis

Paradiopatra capbretonensis

Paradoneis armata

Paradoneis lyra

Paraehlersia ferrugina

Parahesione

Paraheteromastus tenuis

Paralacidonia paradoxa

Paramarphysa longula

Paraninoe

Paraonidae

Paraonis

Parapionosyllis

Parapionosyllis brevicirra

Parapionosyllis cabezali

Parapionosyllis elegans

Parapionosyllis minuta

Paraprionospio pinnata

Parastichopus tremulus
102-103 Parasyllidea

265 Parheteromastus

103 Parophryotrocha

265 Parophryotrocha gesae

103 Parophryotrocha isochaeta

101-102, 113, $181 \quad$ Parophryotrocha rhadina

102 Pectinaria californiensis

101-102, 104-107, 113

101-102, 105-107

104-105, 107, 199

101-102, 105

195-106, 199

104-105

101-102, 105-106

104

195,107

101-102, 105-107

102

164

181, 200, 204-205

151-152, 155

265

151-152

332,336

245

348

116

252

176

205

181

91,98

265

199, 221-222

201, 220, 222

228

165

243,245

200, 203

91,96

82

243, 245

176

181

228,232

219-222, 224

$181,183-184$

181, 183-184

$169,172-173,176-178$

116
Pedinosoma curtum

Pelagobia longicirrata

Perca marina

Perinereis aibuhitensis

Perinereis cultrifera

Perinereis tetradentica

Perinereis vancaurica

Petaloproctus borealis

Petita amphophthalma

Phalacrophorus pictus

Phalacrophorus uniformis

Pholoe balthica

Pholoe synophthalmica

Phragmathopoma caudata

Phyllo kupfferi

Phyllochaetopterus

Phyllochaetopterus socialis

Phyllodoce groenlandica

Phyllodoce laminosa

Phyllodoce rosea

Pilargis verrucosa

Pionosyllis lamelligera

Piromis eruca

Pisione

Pisione parapari

Pisione remota

Pista

Pista atypica

Pista australis

Pista cristata

Pista cristata

Pista curtiuncata

Pista elongata

Pista fasciata

Pista microlobata

Pista robustieta

Pista shizugawaensis

Pista sinusa

Placostegus tridentatus

Plakosyllis brevipes

Platinereis massiliensis

Platynereis dumerilii

Pleijelius longae

Pleijelius n. gen.
165

243-245

151-155

152,155

151-153, 155

151, 154-155

36

212-213

212-213

118

287, 289, 291-292

265

289

289

75-77

348-349

212-214

209, 212-214

265

222

91,92

265

36,44

252-253

265

265

265

199, 201

$220,221,222,228$

265

176

220-222, 224

220-222

139-144

139

144

181

139

144

139

139

139

139

141-144

144

$115,119-120$

222

349

179, 181-184, 204

157-158, 160-163

158-160, 163-164 
Plotohelmis capitata

Podarkeopsis

Podarkeopsis galangaui

Poecilochaetus fauchaldi

Poecilochaetus fulgoris

Polycirrinae

Polydora

Polydora cornuta

Polydora ligni

Polygordius appendiculatus

Polygordius lacteus

Polyophthalmus pictus

Polysiphonia opaca

Pomatoceros

Pomatoceros lamarcki

Pomatoceros triqueter

Pontodora pelagica

Posidonia oceanica

Primnoa resedaeformis

Prionospio

Prionospio cornuta

Prionospio delta

Prionospio fallax

Prionospio peruana

Prionospio pulcra

Proceraea aurantiaca

Proceraea picta

Procerastea nematodes

Protoariciella uncinata

Protodorvillea kefersteini

Protodorvillea wui

Psamathe fusca

Pseudocapitella incerta

Pseudopolydora

Pterosiphonia complanata

Rhodine loveni

Rhynchonerella angelini

Rhynchonerella petersi

Rullierinereis

Rullierinereis ancornunezi

Rullierinereis auxiliadorae

Rullierinereis bahamensis

Rullierinereis tenerifensis

Rullierinereis zebra

Sabella pavonina

Sabella spallanzanii

Sabellaria alveolata

Sabellaria bellis

Sabellaria spinulosa
211,213

164

199

199

265

269-270, 276

181

200, 203, 204, 205, 265

349

219-220, 222

220-222

$179,181-184$

181

220

221-222

$115,120,221-222$

212-213

199, 206, 301-303, 307-308

116

326

243

48

200, 204, 219-222

$169,172-173,176-177$

221-222, 224

228

228

228-229

$187,191,193$

222

172,176

265

265

221,222

227

205, 265

211,213

212-213

$145,147,149-150$

$145,147,149$

149

149

149

149

266

$280,284,286$

265

$249,252,255$

201,227
Sabellastarte spectabilis

279-281, 284-285

Sabellidae 181, 279-280, 286, 319, 321, 324-325

Sabellides octocirrata

Sagitella kowalewski

Salvatoria clavata

Salvatoria limbata

Schistomeringos

Schistomeringos rudolphi

Scionella

Scolaricia typica

Scolelepis matsugae

Scolelepis quinquedentata

Scoletoma impatiens

Scoloplos (Leodamas) gracilis

Scoloplos armiger

Serpula

Serpula norvegica

Serpula tridentata

Serpula triquetera

Serpula triqvetra

Serpulidae

Sigalion cirriferum

Sigambra tentaculata

Sinohesione

Sipunculus nudus

Sirsoe

Sphaerosyllis

Sphaerosyllis austriaca

Sphaerosyllis cryptica

Sphaerosyllis hystrix

Sphaerosyllis pirifera

Sphaerosyllis taylori

Spio caudatus

Spio decoratus

Spiochaetopterus

Spiochaetopterus costarum

Spionidae

$59,61,63-64,181,243$, $245,319,321,324-325,328$

Spiophanes bombyx

$176,219-222$

Spiophanes kroyeri reyssi

199

Spiophanes soderstroemi

172,176

Spirorbis spirorbis

265

Squalus maximus

116

Sternaspis scutata

$200,201,204$

Streblospio gynobranchiata

Streptosyllis websteri

201, 203, 204, 205

219-222, 265

Strongilocentrotus droebachiensis $\quad 314-315,317$

Strongilocentrotus franciscanus

314,317

181

Stypocaulon scoparium

Syllidae

Syllides convolutus
$9,11,13,15-16,181$

181 
Syllides fulvus

Syllidia

Syllinae

Syllis

Syllis amica

Syllis armillaris

Syllis beneliahuae

Syllis bouvieri

Syllis columbretensis

Syllis corallicola

Syllis crassicirrata

Syllis garciai

Syllis gerlachi

Syllis gracilis

Syllis hyalina

Syllis krohnii

Syllis marugani

Syllis nipponica

Syllis okaday

Syllis prolifera

Syllis prolixa

Syllis setoensis

Syllis truncata criptica

Syllis variegata

Syllis vivipara

Terebella lapidaria

Terebellidae

Terebellides

Terebellides lanai

Terebellides stroemi

Terebellinae

Terpsichore

Tharyx

Tharyx acutus

Tharyx kirkegaardi

Tharyx longisetosa

Thelepodinae
181, 183-184

$228,230,232$

225, 228-234

228

181-184

228

228

15

201, 204, 228

228

225, 228-234

228

$14-15,228$

9, 12-15

15

15

179, 181-184, 228-230, 232-234

187, 191, 193-194

14

181

$181,225,228-231,233$

228, 232

265

181, 269-271, 273-275

273, 275-276

$249,252,255$

266

269-270, 276

104

$46,72-73$

72

46

$169,172-175$

269-270
Thelepus

Tomopteris elegans

Tomopteris nationalis

Tomopteris planktonis

Trachytrypane jeffreysii

Travisia

Travisia lithophila

Travisia amadoi

Travisia antarctica

Travisia forbesi

Travisia glandulosa

Travisia kerguelensis

Travisia kerguelensis gravieri

Travisia monroi

Travisia nigrocincta

Travisia olens

Travisia palmeri

Travisia profundi

Travisia tincta

Travisiinae

Travisiopsis dubia

Trichobranchinae

Trichobranchus

Trypanosyllis coeliaca

Trypanosyllis zebra

Typhloscolex mulleri

Typosyllis amica

Typosyllis pulchra

Urosiphon

Veneriserva

Vermiliopsis striaticeps

Wesenbergia

Zostera marina
104

155

164

206

273

212-213

209, 212-214

212-213

265

101-103, 108-113

109

$249,252,255-256$

101,109

108,265

109

101-102, 109

101-102, 109-111

101-102, 110

109

109

101-102, 111 109

101-102, 112

102,113

209, 212-213

269-270, 275

273

228

228

213

265

348-349

220 